\title{
CAUSALIDADE E MECANISMOS EM CiÊnCIA Política
}

\section{Dalson Britto Figueiredo Filho ${ }^{1}$, Enivaldo Carvalho da Rocha ${ }^{2}$, José Alexandre da Silva Junior ${ }^{3}$ e Ranulfo Paranhos ${ }^{4}$}

\begin{abstract}
RESUMO
Esse artigo defende que os desenhos de empíricos de pesquisa em Ciência Política devem ser formatados com o objetivo de produzir inferências causais falsificáveis. Inferir no sentido de utilizar informações disponíveis para apresentar conclusões a respeito de informações indisponíveis. Causais de modo que a ocorrência de $x$ altere a probabilidade de ocorrência de y. E falsificáveis no sentido de que a inferência pode ser demonstrada falsa por um desenho de pesquisa concorrente. Além disso, as explicações devem identificar o mecanismo causal que conecta variável dependente e variável independente.
\end{abstract}

Palavras-chaves: Causalidade. Mecanismos. Inferência. Desenho de pesquisa.

\section{CAUSAlity AND MECHANISMS In POLITICAL SCIENCE}

ABSTRACT

This paper claims that Political Science's empirical research designs should be framed in order to produce falsifiable causal inferences. Inferring in the sense of utilizing available information in order to present conclusions about unavailable ones. Causal so that the occurrence of $x$ alters the probability of the occurrence of $y$. And falsifiable in the sense

\footnotetext{
1 Pós-Doutorando em Ciência Política na universidade Federal de Pernambuco, Brasil. dalsonbritto@yahoo.com.br.

2 Professor do Departamento de Ciência Política da Universidade Federal de Pernambuco, Brasil. enivaldocrocha@gmail.com.

${ }^{3}$ Professor da Universidade Federal de Alagoas, Brasil. jasiunior2007@yahoo.com.br.

${ }^{4}$ Professor da Universidade Federal de Alagoas, Brasil. Brasil. ranulfoparanhos@me.com
} 
that the inference can be shown to be false by a rival research design. Moreover, the explanations should identify the causal mechanism that links explanans and explanandum.

Keywords: causality; mechanisms; inference; research design.

Methodology, not ideology, defines the most important cleavages within the social sciences today.

John Gerring

\section{INTRODUÇÃO}

competição entre paradigmas é um elemento central para entender as revoluções
científicas, ou seja, "aqueles episódios de desenvolvimento não-cumulativo, nos incompatível com o anterior" (KUHN, 1975, p. 125). Pela própria natureza das Ciências Sociais, essas revoluções são mais restritas. Isso porque a Ciência Social convive com uma pluralidade de paradigmas que orientam a atividade da comunidade científica em sentidos distintos e, às vezes, antagônicos. Além disso, o processo de acumulação do conhecimento em Ciências Humanas é menos sistemático do que nas Ciências Naturais. No entanto, é impossível negar a existência de um debate não só sobre o conceito de Ciência Social, mas também a respeito dos métodos e técnicas mais apropriados à investigação dos fenômenos sociais.

Para Gerring (2001), ciência social "é o estudo científico das ações humanas que foca em elementos de pensamento e comportamento não biológicos" (GERRING, 2001, p. $v x)$. E qual é o seu principal objetivo? King, Keohane e Verba (1994) afirmam que "o cientista social deve realizar inferências descritivas ou causais, procurando por explicações e generalizações" (KING; KEOHANE; VERBA, 1994, p. 188). Estes autores, a pesquisa social, se quantitativa ou qualitativa, tem dois principais objetivos: (a) descrever e (b) explicar. Ambos são essenciais. É impossível construir explicações causais inteligíveis sem um bom trabalho descritivo. A descrição, por sua vez, perde a maior parte do sentido se não estiver a serviço da elaboração de explicações causais. Fundamentalmente, os autores afirmam que não é a briga entre descrição x explicação que define o que é conhecimento científico. Mas sim se inferências sistemáticas podem ser realizadas a partir de procedimentos válidos. A inferência, se descritiva ou causal, quantitativa ou qualitativa, é o principal objetivo da ciência. A inferência descritiva é 0 processo de compreensão de fenômenos não observáveis a partir de um conjunto de observações (KING; KEOHANE; VERBA, 1994, p. 55). A inferência causal, por sua vez, 
requer a introdução de uma ou mais variáveis independentes para explicar a variação de uma determinada variável dependente. 0 tipo de resposta ofertada pelo pesquisador a essas questões o aproximará de uma ou outra corrente paradigmática. Por sua vez, a filiação a um determinado paradigma tende a limitar tanto as modalidades de questionamento quanto os meios utilizados para investigar os fenômenos sociais (MARSH; FURLONG, 2002).

Nosso público alvo são estudantes de graduação e pós-graduação em Ciência Política e nossa principal meta é estabelecer um modelo básico de formatação de desenhos de pesquisa que garanta a replicabilidade dos resultados reportados. Acreditamos que 0 padrão de replicabilidade fornece três principais vantagens ao desenvolvimento do conhecimento científico: (1) substantiva, na medida em que contribui para o aprimoramento e acúmulo do conhecimento científico; (2) pedagógica, já que facilita a compreensão de noções básicas de análise de dados e (3) transparência, na medida em que protege a comunidade acadêmica não só contra erros honestos, como também de fraudes intencionais. Fundamentalmente, esse artigo defende que os desenhos empíricos de pesquisa em Ciência Política devem ser formatados com 0 objetivo de produzir inferências causais falsificáveis. Inferir no sentido de utilizar informações disponíveis para apresentar conclusões a respeito de informações indisponíveis. Causais de modo que a ocorrência de $x$ altere a probabilidade de ocorrência de $y$. E falsificáveis no sentido de que a inferência pode ser demonstrada falsa por um desenho de pesquisa concorrente. Além disso, as explicações devem identificar 0 mecanismo causal que conecta variável dependente e independente.

Na primeira seção do artigo se discutem os conceitos de ontologia, epistemologia e metodologia. $\mathrm{Na}$ segunda parte se revisam as principais características de três diferentes correntes paradigmáticas: a) positivismo; b) hermenêutica (relativismo) e c) realismo. Na terceira seção se explora o problema da causalidade, na quarta parte se analisa a importância da explicação por mecanismos e, na última seção, apresentam-se as principais conclusões.

\section{ONTOLOGIA, EPISTEMOLOGIA E METODOLOGIA}

Para Marsh e Furlong (2002, p. 18-19), "a posição ontológica reflete a visão do pesquisador a respeito da natureza do mundo, sua posição epistemológica reflete sua visão a respeito do que se pode conhecer sobre o mundo e como". A ontologia é anterior e se preocupa com o estudo do ser, ou seja, a posição ontológica reflete a visão do pesquisador sobre o que pode ser conhecido. Os fundacionistas/essencialistas acreditam que o mundo existe de forma independente e que é possível conhecê-lo. Por outro lado, a posição antifundacionista defende que os fenômenos observados são socialmente 
construídos e nega a possibilidade do conhecimento objetivo da realidade, argumentando que o conhecimento é necessariamente mediado pela subjetividade.

A epistemologia, por sua vez, refere-se a como se pode conhecer a realidade, ou seja, a epistemologia é uma teoria do conhecimento. A questão central a ser respondida é se 0 pesquisador pode observar relações objetivas entre os fenômenos sociais. Em caso afirmativo, como o pesquisador pode identificar essas relações? Para Marsh e Furlong (2002), o tipo de resposta que o pesquisador oferece a essas questões informa a sua posição epistemológica. Por fim, a metodologia diz respeito aos métodos e técnicas empregados para investigar os fenômenos. Para os autores, as posições ontológicas e epistemológicas definem como o pesquisador vai utilizar os métodos e as técnicas de pesquisa na investigação dos fenômenos sociais.

Della Porta e Keating (2008) afirmam que, teoricamente, ontologia, epistemologia e metodologia são eventos independentes. No entanto, eles tendem a interagir já que a posição ontológica e epistemológica do pesquisador o aproximará de um ou outro tipo de método e/ou técnica. Isso quer dizer que a metodologia utilizada para investigar os fenômenos sociais é uma consequência do seu paradigma epistemológico. Para os propósitos desse trabalho, é importante analisar as principais características de três diferentes correntes epistemológicas: (1) positivista; (2) hermenêutica e (3) realista.

\section{Positivismo}

Zald (1995, p. 456) define positivismo como uma posição epistemológica que procura realizar generalizações universais, ou leis, a partir da observação objetiva da realidade. Della Porta e Keating (2008) argumentam que para a concepção positivista:

[...] o mundo existe de forma objetiva, de forma independente do pesquisador, sendo possível conhecer integralmente a realidade. 0 objetivo do pesquisador é descrever e analisar essa realidade [...] assim como nas ciências naturais, existem regras sistemáticas e regularidades associadas ao objeto de estudo (DELLA PORTA; KEATING, 2008, p. 23).

Em termos ontológicos, o positivismo é fundacionista - o mundo/realidade existe de forma independente do nosso conhecimento. Tanto a observação direta dos fenômenos quanto a busca por relações causais são enfatizadas. Além disso, acredita-se na objetividade do conhecimento e na possibilidade de distinguir questões positivas de questões normativas. A partir da observação sistemática de fenômenos é possível formular leis gerais válidas no tempo e no espaço. No que diz respeito às estruturas complexas não observáveis, os positivistas acreditam que essas estruturas não devem ser 
consideradas na construção do conhecimento científico já que a sua incorporação aos modelos explicativos tendem a tornar as proposições não falsificáveis. Seguindo a definição popperiana, se uma proposição não é falsificável ela não pode ser considerada como pertencente ao ramo do conhecimento científico.

Resumindo, é possível afirmar que o positivismo pode ser compreendido a partir das seguintes características: a produção do conhecimento passa necessariamente pela observação de fenômenos empíricos, mensuração e teste de hipóteses. 0 objetivo do conhecimento científico é identificar as causas que explicam os fenômenos sociais. Parte-se do pressuposto de que esses fenômenos não são aleatórios, sendo possível identificar relações causais entre variáveis. Os métodos e técnicas empregados na investigação da realidade devem ser independentes dos valores subjetivos do pesquisador (objetividade do conhecimento). Dessa forma, os métodos e técnicas utilizados pelas ciências naturais podem e devem ser utilizados para investigar os fenômenos sociais ${ }^{5}$.

\section{HERMENÊUTICA (RELATIVISMO)}

Em grande medida, a tradição hermenêutica se desenvolveu como uma crítica ao positivismo. Em diferentes níveis, a tradição relativista rejeita os postulados da ciência positivista, argumentando que as ciências naturais e as ciências sociais constituem abordagens amplamente distintas e, por isso, requerem métodos específicos. Estudar um componente químico em um laboratório em nada se assemelha a investigação dos fenômenos sociais.

Para a posição interpretativista existe uma diferença fundamental entre o mundo natural e o mundo social: o significado. Por isso, a realidade social deve ser interpretada. $\mathrm{Na}$ tradição sociológica alemã o termo verstehen é utilizado como sinônimo de

\footnotetext{
${ }^{5}$ Para os propósitos desse trabalho, é importante destacar também a abordagem comportamentalista. Para Sanders (2002), a abordagem comportamentalista em ciências sociais se preocupa com uma questão extremamente simples: por que as pessoas se comportam de determinada forma? Em termos históricos, o comportamentalismo assumiu um papel importante nas décadas de 1950 e 1960, sendo profundamente influenciado pela obra de Comte e pelo positivismo lógico do círculo de Viena. 0 foco repousa sobre 0 comportamento observável, individual ou agregado (coletivo), e assume-se que a explicação sobre 0 comportamento deve ser passível de teste empírico. Comparativamente, o comportamentalismo se aproxima mais do positivismo do que do realismo ou da hermenêutica. Por exemplo, assim como os positivistas, a abordagem comportamentalista defende o falsificacionismo como um elemento central na avaliação de teorias. Criticamente, uma das principais objeções dirigidas ao behaviorismo refere-se à tendência de estudar aqueles fenômenos que são mais facilmente mensuráveis em detrimento daqueles que são teoricamente importantes. Um dos efeitos negativos da ênfase em investigar os fenômenos diretamente observáveis e, por isso, de mais fácil mensuração, seria distanciar pesquisador de fenômenos substantivamente importantes, como a corrupção, por exemplo, mas que não podem ser diretamente observados nem tampouco facilmente medidos.
} 
compreensão/interpretação. É nesse sentido que a abordagem hermenêutica tem no significado subjetivo das ações o seu "core" (MARSH; FURLONG, 2002, p. 24).

Em termos ontológicos, a posição interpretativista é antifundacionista e considera que é impossivel compreender os eventos históricos e/ou os fenômenos sociais sem analisar as percepções que os indivíduos têm do mundo (MARSH; FURLONG, 2002, p. 24-25). A busca por relações causais é desprezada e se questiona a possibilidade de produzir conhecimento de forma objetiva já que não só o objeto a ser estudado, mas também o pesquisador, estão imersos na mesma construção social.

Os relativistas se aproximam daquilo que Tilly (2001) denominou de visão cética. Para os céticos, os fenômenos políticos são considerados demasiadamente complexos e contingentes. 0 pesquisador pode tentar reconstruir as experiências dos atores, descrever, interpretar e conferir significado subjetivo às ações, mas não existe preocupação em explicar sistematicamente os fenômenos sociais e políticos. A conclusão é bastante direta: a busca por explicações causais é estéril. Ao invés disso, as Ciências Sociais devem se ocupar com a interpretação das ações dos atores sociais, buscando revelar os seus significados subjetivos.

Em síntese, o relativismo pode ser compreendido a partir das seguintes características: os fenômenos são socialmente construídos de modo que elementos observados em uma sociedade $X$ podem estar ausentes em uma sociedade $Z$, não sendo possivel identificar regularidades entre os fenômenos. Como os atores sociais produzem a realidade social, a busca por relações de causa e efeito é inapropriada. 0 foco deve ser interpretar e compreender as experiências subjetivas dos atores sociais, seus sentimentos, suas estruturas de pensamento e seu comportamento. Os métodos e técnicas utilizados para compreender essa realidade devem refletir o fato de que a realidade social é relacional, subjetiva e socialmente construída.

\section{REALISMO}

0 realismo adota uma ontologia fundacionista e defende que a chave do conhecimento repousa sobre estruturas complexas não observáveis. Della Porta e Keating (2008, p. 24) afirmam que existem mecanismos governando a realidade social que podem não ser diretamente observáveis, mas nem por isso devem ser desprezados. De acordo com os realistas, o objetivo do conhecimento científico é identificar as estruturas causais latentes que explicam as regularidades empiricamente observáveis.

Além disso, os realistas acreditam que a observação direta de fenômenos, desde que seja devidamente orientada pela teoria, é um importante procedimento no processo de construção do conhecimento. Não existe no realismo a separação rígida (como há no 
positivismo) entre teoria e observações, ou seja, para os realistas toda observação é dependente da teoria.

0 realismo também considera importante a busca por relações causais (aqui se aproxima dos positivistas), mas não concebe a distinção entre questões positivas e questões normativas (aqui se aproxima dos relativistas). Em particular, alguns realistas preferem utilizar o termo configuração.

Em síntese, o realismo pode ser compreendido a partir das seguintes características: as estruturas complexas não observáveis constituem um elemento central para compreender os fenômenos sociais. 0 objetivo do conhecimento é desvendar os mecanismos causais produzidos por essas estruturas complexas não observáveis, analisando como eles influenciam os fenômenos sociais. 0 quadro abaixo sintetiza as principais características de cada corrente.

Quadro 1 - Principais características do Positivismo, da Hermenêutica e do Realismo

\begin{tabular}{|l|l|l|l|l|}
\hline Corrente & Ontologia & Foco & Relações causais & Objetividade \\
\hline Positivista & Fundacionista & $\begin{array}{l}\text { Relações causais e modelos } \\
\text { preditivos }\end{array}$ & Sim & Sim \\
\hline Hermenêutica & $\begin{array}{l}\text { Anti- } \\
\text { fundacionista }\end{array}$ & Significado e compreensão & Não & Não \\
\hline Realista & Fundacionista & $\begin{array}{l}\text { Estruturas complexas não } \\
\text { observáveis }\end{array}$ & Sim & $\begin{array}{l}\text { Observação } \\
\text { mediada pela } \\
\text { teoria }\end{array}$ \\
\hline
\end{tabular}

Fonte: Adaptado de Marsh e Furlong (2002).

Como observa Gerring (2001), o curioso é que as Ciências Sociais recebem críticas de todos os lados. Para os positivistas, elas carecem de maturidade e a relação com as humanidades é entendida como deletéria. Ou seja, tem-se pouca ciência na Ciência Social. Fundamentalmente,

[...] o modelo das ciências naturais quando aplicado às ciências sociais é inadequado dada a imprecisão, inconsistência e indeterminação das humanidades (ao analisar o mesmo fenômeno, cinco pesquisadores irão produzir cinco diferentes explicações), falta de progresso e cumulatividade, teoria incipiente, falta de objetividade e neutralidade axiológica (GERRING, 2001, p. xii).

Para os humanistas (relativistas), observa-se exatamente o contrário. A Ciência Social perdeu seu sentido quando começou a analisar os fenômenos sociais emulando os 
métodos das Ciências Naturais, ou seja, tem-se ciência demais na Ciência Social. Comparativamente, o realismo parece adotar uma posição mais equilibrada na medida em que comunga algumas características do positivismo, mas também partilha a preocupação com elementos do relativismo.

Na próxima seção se discutem, mais detalhadamente, dois elementos centrais dentro desse debate: a) a possibilidade de relações causais entre os fenômenos sociais e b) as vantagens e limitações da explicação por mecanismos. abordam essas questões.

\section{O PROBLEMA DA CAUSALIDADE}

Lexicamente, tanto Blalock (1967) quanto Van Evera (1997) introduzem dois vocábulos centrais para entender as modernas definições de causalidade: variável e efeito. 0 conceito de variável refere-se a um atributo, direta ou indiretamente observável, sujeito a variação quantitativa ou qualitativa. 0 efeito, por sua vez, é uma medida do impacto de $x$ (variável independente) sobre $y$ (variável dependente). Para King, Keohane e Verba (1994, p. 81), efeito causal é a diferença entre os componentes sistemáticos das observações realizadas quando as variáveis explicativas assumem um determinado valor e os componentes sistemáticos de observações comparáveis quando as variáveis explicativas assumem outros valores. Similarmente, para Brady e Collier (2004), efeito causal é a diferença entre valores das variáveis dependentes que é observado de acordo com a variação dos valores das variáveis independentes ${ }^{6}$.

Esse trabalho define causalidade como a diferença entre os valores assumidos pela variável dependente a partir da variação dos valores da variável independente, de tal sorte que a ocorrência de $x$ influencia a probabilidade da ocorrência de $y$.

Pearl (2000) aponta três principais pressupostos para identificar a presença de uma relação causal: (1) associação entre as variáveis (correlação) ${ }^{7}$; (2) precedência temporal e (3) não-espuriosidade da relação. A figura abaixo ilustra esses pressupostos ${ }^{8}$.

\footnotetext{
${ }^{6}$ Para os leitores interessados nesse debate ver Rubin (1974) e Holland (1986).

${ }^{7}$ Correlação não é causalidade: esse é um dos principais mantras da Estatística e por consequência das disciplinas que utilizam modelos e técnicas estatísticas em seus desenhos de pesquisa. Basicamente, toda relação de causalidade pressupõe correlação entre as variáveis de interesse do pesquisador, mas a simples presença de correlação entre x e y não implica uma relação causal.

${ }^{8}$ Stuart Mill identificou três elementos para estabelecer uma relação causal: (1) a causa precede o efeito; (2) a causa deve estar correlacionada com o efeito e (3) não é possível encontrar nenhuma explicação adicional para explicar o efeito.
} 
Figura 1 - Pressupostos da causalidade

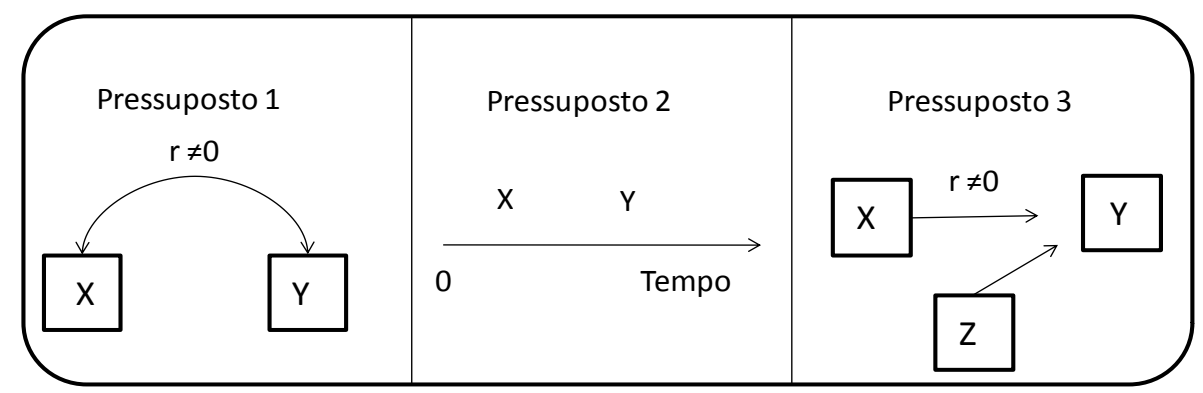

Fonte: Pearl (2000).

A primeira condição, associação entre as variáveis, sugere que a ocorrência de $y$ não é independente da ocorrência de $x$. Duas variáveis são independentes quando a variação na magnitude de uma delas não apresenta nenhuma associação com a variação na magnitude da outra. Dito de outra forma, considera-se que $x$ e $y$ são independentes quando a distribuição condicional de $y$ não varia de acordo com a distribuição de $x$. Logo, o primeiro pressuposto para identificar a presença de uma relação causal é a existência de correlação entre $x$ e $y(r \neq 0)$.

A segunda condição, precedência temporal, é intuitiva já que o que aconteceu depois não pode causar 0 que aconteceu antes. Para considerar $x$ como causa de $y$ é necessário que a ocorrência de $x$ preceda a ocorrência de $y$. Por exemplo, um indivíduo é alvejado $(x)$ e depois vem a falecer $(y)$. Infere-se que a morte do indivíduo $(y)$ é explicada pelo evento anterior $(x)$, sendo impossível observar a ocorrência de $y$ antes da ocorrência de $x$. Dessa forma, o segundo pressuposto para identificar uma relação causal é a assimetria temporal entre $x$ e $y$.

A terceira condição, não-espuriosidade da relação, exige a eliminação de causas concorrentes. Suponha que um indivíduo é alvejado $(x)$, mas também é envenenado $(z)$ e depois vem a óbito $(y)$. Para que $x$ possa ser considerado causa de $y$ é necessário que 0 seu efeito seja observado, independente da presença de $z$.

Gerring (2001) sugere critérios bastante semelhantes, mas adiciona um elemento. 0 quadro abaixo sumariza essas informações. 
Quadro 2 - Relação causal (critérios adicionais)

\begin{tabular}{|l|l|}
\hline Critério & Definição \\
\hline Diferenciação (exogeneidade) & $\begin{array}{l}\text { Para que } x \text { possa ser considerado causa de } y \text {, é necessário diferenciar } \\
x \text { de } y \text {. Ou seja, a causa deve se diferenciar lógica e empiricamente da } \\
\text { consequência a ser explicada. }\end{array}$ \\
\hline $\begin{array}{l}\text { Antecedência temporal } \\
\text { (exogeneidade) (priority) }\end{array}$ & $\begin{array}{l}\text { Para que } x \text { possa ser considerado causa de } y \text {, a ocorrência temporal } \\
\text { de } x \text { deve preceder a ocorrência temporal de } y \text { ( } x \text { deve ocorrer antes de } \\
y \text { ). }\end{array}$ \\
\hline $\begin{array}{l}\text { Independência (exogeneidade, } \\
\text { assimetria, recursividade) }\end{array}$ & $\begin{array}{l}\text { Para que } x \text { possa ser considerado causa de } y \text {, a ocorrência de } x \text { deve } \\
\text { ser independente da ocorrência de } y \text { ( } y \text { não pode influenciar a } \\
\text { probabilidade da ocorrência de } x \text { ). }\end{array}$ \\
\hline Contingência & $\begin{array}{l}\text { Para que } x \text { possa ser considerado causa de } y, x \text { não pode ser } \\
\text { contingente de } z \text { (deve-se excluir causas concorrentes). }\end{array}$ \\
\hline
\end{tabular}

Fonte: Adaptado de Gerring (2001, p. 138).

0 critério de diferenciação entre $x$ e $y$ parece ser evidente, ou seja, para que $x$ possa ser considerado causa de $y$ deve existir uma distinção lógica e empírica entre ambos. 0 critério da antecedência temporal elencado por Gerring (2001) é o mesmo do pressuposto 2 identificado por Pearl (2000): $x$ deve ocorrer antes de $y$. Aparentemente, 0 critério da independência (exogeneidade) também parece ser evidente. No entanto, problemas de endogeneidade tem se demonstrado amplamente recorrentes na estimação de modelos empíricos que buscam estabelecer relações causais. 0 quarto critério diz respeito à contingência da relação entre $x$ e $y$, equivalendo o pressuposto 3 elencado por Pearl (2000).

Depois de definir o conceito e os critérios de identificação de uma relação causal, o próximo passo é identificar os tipos de causalidade. A figura abaixo ilustra essas informações. 
Figura 2 - Tipos de causalidade

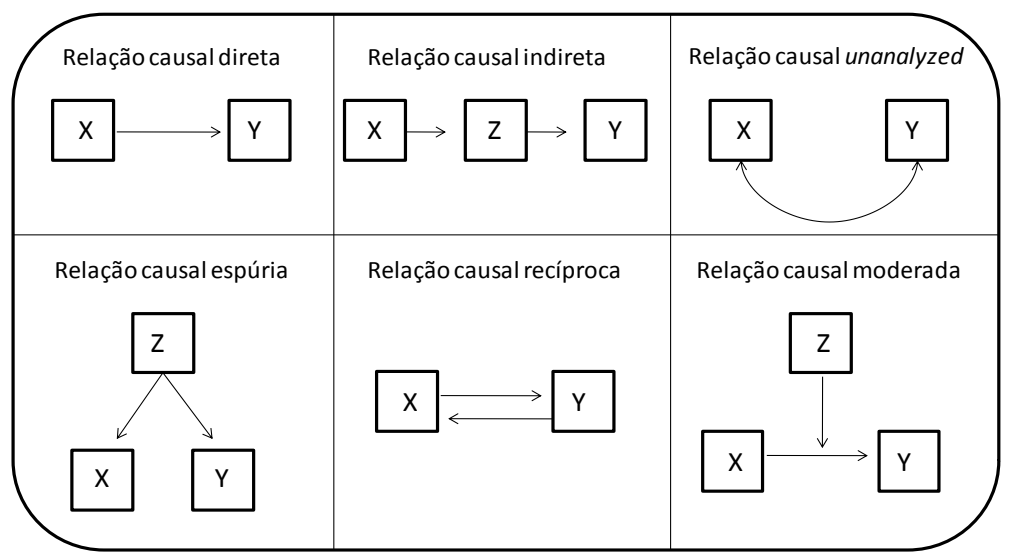

Fonte: Pearl (2000).

Em geral, os cientistas sociais formulam suas hipóteses no sentido de identificar relações causais diretas $(X \rightarrow Y)$. 0 objetivo é estimar em que medida um conjunto de variáveis independentes $\left(x_{n}\right)$ pode ser utilizado para explicar a variação de $y$ (variável dependente). No entanto, dada a complexidade dos fenômenos sociais, é comum observar diferentes tipos de relações causais.

No caso da causalidade indireta, o pesquisador deve examinar se $x$ pode ser considerada uma causa independente de $y$, ou se não está condicionada a uma terceira variável, $z$. Nesse caso, $z$ é considerada variável interveniente. Imagine a relação entre gasto com propaganda tabagista $(x)$, venda de cigarros $(z)$ e incidência de câncer $(y)$. Não faz sentido estabelecer uma relação direita entre investimento em propaganda $(x)$ e incidência do câncer $(y)$, a cadeia causal apenas pode ser reconstruída com a inclusão da venda de cigarros $(z)$. 0 pesquisador espera que quanto maior $x$, maior $z$. E, quanto maior $z$, maior $y$. Logo, enquanto $z$ exerce um efeito direito sobre $y, x$ exerce apenas um efeito indireto.

Na causalidade moderada, o pesquisador procura estimar o impacto que $z$ exerce sobre 0 efeito de $x$ sobre $y^{9}$, são os chamados efeitos interativos ${ }^{10}$. Por exemplo, ao se

\footnotetext{
${ }^{9}$ Considere o seguinte modelo, $Y=\alpha+\beta 1 \mathrm{X} 1+\beta 2 \mathrm{X} 2+\varepsilon$

0 pressuposto da aditividade assume que 0 efeito de $X_{1}$ sobre $Y,\left(\beta_{1}\right)$, independe do valor de $X_{2}$. No entanto, dada a complexidade dos fenômenos sociais, é razoável assumir não aditividade ou interação entre as variáveis. No modelo com interação, $Y=\alpha+\beta 1 X 1+\beta 2 X 2+\beta 3 X 1 X 2+\varepsilon$ o termo interativo $\left(X_{1} X_{2}\right)$ captura 0 efeito condicional das variáveis independentes. Nesse modelo, $\beta_{1}$ representa 0 efeito de $X_{1}$ sobre $Y$ quando $X_{2}=0$. Similarmente, $\beta_{2}$ representa 0 efeito de $X_{2}$ sobre $Y$ quando $X_{1}=0$. Para um
} 
investigar o efeito da escolaridade (x) sobre a renda (y) por gênero, o pressuposto da aditividade assume que o efeito da escolaridade é constante para ambos os grupos. No entanto, o pesquisador, teoricamente orientado, sabe que existe preconceito de gênero na sociedade e que as mulheres mesmo com mais escolaridade auferem renda menor. Para captar essa informação, ele deve inserir um termo interativo entre as variáveis independentes (escolaridade e sexo) na equação de regressão.

$\mathrm{Na}$ causalidade espúria, por terem a mesma causa $(z), x$ e $y$ serão correlacionados, mas como o pesquisador não controlou por $z$, ele chega à conclusão de que $x$ é causa de $y$. Considere a relação entre golfar e engordar. Isso porque existe a crença de que bebê que golfa muito, ganha peso mais rápido. No entanto, a correlação observada entre golfar $(x)$ e engordar $(y)$ pode ser explicada na medida em que elas têm a mesma causa: gulodice $(z)$. Ao se controlar pelo efeito da gulodice, a correlação entre as variáveis desaparece, ou seja, a relação entre as variáveis era espúria ${ }^{11}$.

Na causalidade recíproca, $x$ influencia $y$, mas $y$ também influencia $x$, não sendo possível estimar com precisão o efeito de uma variável sobre a outra, violando o critério da exogeneidade estabelecido por Gerring (2001). A modelagem estatística desenvolveu algumas técnicas para lidar com os problemas de endogeneidade. As mais usualmente empregadas são a utilização de variáveis instrumentais e a aplicação de modelos de equações estruturais. 0 modelo de mínimos quadrados ordinários produz estimadores eficientes e não-viesados desde que os pressupostos sejam devidamente respeitados. A necessidade por variáveis instrumentais surge quando alguns desses pressupostos são violados (especificação do modelo ou ausência de erros ou independência).

Em termos técnicos, Blalock (1967) defende que uma das ferramentas para estabelecer relações causais utilizando dados observacionais são os modelos de regressã $0^{12}$. Isso porque essa técnica permite estimar o grau de associação entre $Y$ (variável dependente) e o conjunto de variáveis independentes (explicativas). 0 objetivo é resumir a correlação entre X e Y em termos da direção (positiva e negativa) e

trabalho clássico sobre termos interativos ver Friedrich (1982). Para uma aplicação bastante didática ver Jaccard, Turrisi e Wan (1990) e Brambor, Clark e Golder (2006).

${ }^{10} \mathrm{Um}$ dos pressupostos dos modelos de regressão é o da aditividade, ou seja, de que o efeito de x sobre y é constante para diferentes valores de $\mathrm{z}$.

${ }^{11}$ Esse exemplo tem sido tradicionalmente utilizado pelo professor Jorge Alexandre no curso intensivo de Metodologia Quantitativa (MQ) em Ciências Sociais da Universidade Federal de Minas Gerais (UFMG). De acordo com o Houaiss, o termo gulodice é proveniente da alteração da palavra gulosice e remonta ao século XV. No nordeste brasileiro a palavra gulodice é usualmente utilizada para designar pessoas que comem de forma excessiva.

${ }^{12}$ A maior parte dos estudos empíricos em Ciências Sociais realiza inferências utilizando dados observacionais. Recentemente, a utilização de experimentos e quase-experimentos vem se difundido na Ciência Política. Para os leitores interessados em aprender mais experimentos ver Campbell e Stanley (1966), Dean e Voss (1999), Montgomery (2001), Shadish, Cook e Campbell (2002). 
magnitude. Mais especificamente, é possível utilizar as variáveis independentes para predizer os valores da variável dependente. Em regressões multivariadas é possível também identificar a contribuição de cada variável independente sobre a capacidade preditiva do modelo como um todo ${ }^{13}$. Todavia, não existe nenhuma técnica estatística capaz de detectar, sozinha, causalidade entre $x$ e $y$. É nesse sentido que a preocupação com a causalidade deve ser um componente central dos desenhos de pesquisa em Ciência Política.

\section{A IMPORTÂNCIA DA EXPLICAÇÃO POR MECANISMOS ${ }^{14}$}

Para entender a importância da explicação por mecanismos deve-se definir o que é uma explicação e depois o que é mecanismo. Bunge (1997) identifica quatro tipos gerais de explicação. 0 quadro abaixo sumariza essas informações.

Quadro 3-Tipos de explicação

\begin{tabular}{|l|l|}
\hline Tipo de explicação & Ênfase \\
\hline Covering Law & Derivação de leis gerais a partir de observações particulares \\
\hline Interpretative & Significado e interpretação das ações e fenômenos sociais \\
\hline Functional & Propósito da ação (telos) \\
\hline Mechanismic & Mecanismo responsável pelo resultado observado \\
\hline
\end{tabular}

Fonte: Adaptado de Gerring (2005).

A explicação do tipo covering law consiste na derivação de leis gerais a partir de observações particulares na melhor tradição positivista. A explicação do tipo

\footnotetext{
${ }^{13}$ Para uma introdução intuitiva ao modelo de regressão de mínimos quadrados ordinários ver Figueiredo Filho, Silva Junior e Rocha (2011).

${ }^{14}$ Para os propósitos desse artigo, é importante destacar a diferença entre explicação e previsão. Considere a seguinte afirmação: "o Náutico vai ser rebaixado no Campeonato Brasileiro em 2013". Falsificar previsões é muito mais fácil do que falsificar explicações. Basta esperar o momento da previsão e observar se ela ocorre ou não (GERRING, 2001). Além disso, a previsão pode se confirmar sem existir necessariamente alguma causa subjacente para explicar o resultado observado. Por exemplo, "jumento com testículo suado é preditor de chuva". Mesmo que se observe que choveu depois de que o testículo do jumento ficou suado, estabelecendo covariação e assimetria temporal, é necessário identificar o mecanismo causal que explica o resultado observado. Deixando o exemplo anedótico de lado, suponha que 0 pesquisador esteja interessado em prever 0 resultado de uma eleição. Ele coleta uma amostra aleatória de eleitores, inquirindo sobre a intenção de voto. Por mais acurada que seja o resultado da pesquisa em relação ao resultado oficial, não existe relação causal entre esses dois eventos.
} 
interpretativa enfatiza o significado das ações e dos fenômenos sociais, destacando 0 papel da subjetividade. A explicação funcional enfoca no propósito (telos) da ação, ou seja, representativa dos modelos explicativos de inspiração parsoniana. Por fim, a explicação por mecanismos procura identificar o elo entre inputs e outputs, ou seja, entre variáveis independentes e dependentes.

De acordo com Salmon (1984), explicar um determinado evento significa identificar a causa e, em muitos casos pelo menos, desvendar a relação causal entre a causa e 0 evento que será explicado. Sanders (2002) identifica a necessidade de um elemento causal e a especificação de condições suficientes e necessárias que sejam anteriores ao fenômeno a ser explicado. Brady e Collier (2004) definem explicação como uma afirmação a respeito do porque um determinado fenômeno ocorreu.

Esse trabalho adota a definição Elsteriana que enfatiza o papel dos mecanismos como componentes essenciais da explicação causal. Mas o que é um mecanismo afinal? Merton (1968) define mecanismos como "processos sociais que afetam partes específicas da estrutura social." Tem-se então os conceitos de processo, efeito (consequências) e estrutura social. Para Bunge (1997, p. 410), "processo é um sistema concreto capaz de incentivar ou obstruir alguma mudança no sistema como um todo ou em partes específicas". Para Tilly (2001, p. 25-26), "determinada classe de eventos que alteram as relações entre elementos específicos de forma idêntica ou fortemente similar em diferentes situações". Para Schelling, um mecanismo pode ser definido como um conjunto sistemático de afirmações que oferece uma explicação plausível de como I e 0 estão relacionados (HEDSTROM; SWEDBERG, 1998, p. 5). Assim, mecanismo causal é uma entidade não observada que, quando ativada, gera um resultado de interesse, os mecanismos causais são relações postuladas que o pesquisador imagina que existam (MAHONEY, 2001, p. 580).

Nesse trabalho, adota-se a uma definição de mecanismo que combina as noções de Hedstrom e Swedberg (1998) e Mahoney (2001), leia-se: mecanismos são processos não observáveis que convertem inputs em outputs. 0 quadro 4 sumariza diferentes concepções de mecanismos.

Quadro 4-Definição de Mecanismos

\begin{tabular}{|l|l|}
\hline Autor (ano) & Definição \\
\hline Merton (1968) & Processos sociais que afetam partes específicas da estrutura social \\
\hline Bunge (1990) & $\begin{array}{l}\text { Processo em um sistema concreto capaz de incentivar ou obstruir alguma } \\
\text { mudança no sistema como um todo ou em partes específicas }\end{array}$ \\
\hline Stinchcombe (1991) & Partes de teoria que conectam fenômenos em diferentes níveis de análise \\
\hline
\end{tabular}




\begin{tabular}{|l|l|}
\hline Tilly (1991) & $\begin{array}{l}\text { Determinada classe de eventos que alteram as relações entre elementos } \\
\text { específicos de forma idêntica ou fortemente similar em diferentes situações }\end{array}$ \\
\hline $\begin{array}{l}\text { Hedstrom e Swedberg } \\
\text { (1998) }\end{array}$ & Processos que convertem inputs em outputs \\
\hline Schelling (1998) & $\begin{array}{l}\text { Conjunto de afirmações que fornecem um relato plausível de como inputs e } \\
\text { outputs estão ligados uns aos outros. }\end{array}$ \\
\hline Mahoney (2001) & Entidade não observada que quando ativada gera um resultado de interesse \\
\hline
\end{tabular}

Fonte: 0 autor.

Uma vantagem analítica associada à utilização de mecanismos causais é a sua relação com a ação, sua precisão, sua abstração de contextos concretos e sua estratégia reducionista de abrir a caixa preta (KITSCHELT, 2003, p. 60). Outra vantagem é de que a explicação por mecanismos aumenta a chance do pesquisador diferenciar uma autêntica explicação causal de uma associação acidental, elevando a compreensão do por que nós observamos o que foi observado (HEDSTROM; SWEDBERG, 1998). De acordo com Elster, compreender os detalhes de uma cadeia causal reduz a probabilidade de produzir explicações espúrias em que o pesquisador confunde correlação com causalidade.

Da mesma forma que uma explicação causal sem mecanismos parece incompleta (ELSTER, 1989), uma descrição dos mecanismos que não incorpore a covariação entre variáveis independentes e dependente parece fútil (GERRING, 2005). Bunge (1997) argumenta que se nós desejamos de fato compreender um fenômeno nós precisamos entender como ele funciona. A explicação por mecanismos fornece essa possibilidade analítica a partir do momento que 0 pesquisador pode identificar 0 mecanismo causal que conecta variável dependente e variável independente.

\section{CONSIDERAÇÕES FINAIS}

Para além da produção de artigos científicos, a principal função do conhecimento é melhorar a qualidade de vida das pessoas. Nas sociedades que apresentam problemas sociais intensos, é imprescindível que a produção do conhecimento possa, direta ou indiretamente, beneficiar a população. Acreditamos fortemente que a formatação de desenhos de pesquisa a partir de inferências causais falsificáveis favorece tanto a replicabilidade dos resultados observados quanto a aplicação prática do conhecimento produzido.

Para Abraham Maslow, se a única ferramenta disponível é um martelo, é tentador tratar tudo como se fosse um prego. Portanto, é importante que o pesquisador avalie qual corrente paradigmática é mais adequada para informar a sua atividade de 
pesquisa. Partindo do pressuposto de que toda escolha é trágica, ou seja, cada opção ontológica, epistemológica, teórica e metodológica vai trazer em si vantagens e limitações inerentes a cada opção, um dos principais desafios impostos aos estudiosos é lidar, da melhor forma possível, com esses diferentes tradeoffs. Para isso, é necessário que o pesquisador conheça as potencialidades e os obstáculos associados a cada tipo de percurso. Dessa forma, ele estará mais capacitado a investigar os fenômenos que the interessa, contribuindo para a construção do conhecimento. Talvez, pior do que a tentação de tratar todo problema como um prego, é a incapacidade de distinguir o martelo do prego.

Nesse trabalho, partimos do pressuposto de que o principal objetivo da ciência é explicar a realidade. Por isso, defendemos que os desenhos empíricos de pesquisa em Ciência Política devem ser formatados com o objetivo de produzir inferências causais falsificáveis. Inferir no sentido de utilizar fatos/informações disponíveis para concluir a respeito de fato/informações indisponíveis. Causais de modo de que a ocorrência de $x$ altere a probabilidade de ocorrência de $y$. E falsificáveis de sorte que a qualquer momento a inferência causal pode ser demonstrada falsa por outro desenho de pesquisa concorrente. Por fim, a explicação deve identificar o mecanismo causal responsável por converter inputs em outputs.

\section{REFERÊNCIAS}

BLALOCK, Hubert M. Causal inferences, closed populations, and measures of association. American Political Science Review, Baltimore, v. 61, n. 1, p. 130-136, 1967.

BRADY, Henry; COLLIER, David (Org.). Rethinking social inquiry: diverse tools, shared standards. Lanham: Rowman \& Littlefield, 2004.

BRAMBOR, Thomas; CLARK, William R.; GOLDER Matt. Understanding interaction models: Improving empirical analyses. Political Analysis, 0xford, v. 14, n. 1, p. 63-82, 2006.

BUNGE, Mario. Mechanism and explanation. Philosophy of the Social Science, New York, v. 27, n. 4, p. 410-465, 1997.

CAMPBELL, Donald; STANELY, Julian. Experimental and quasi-experimental designs for research. Chicago, Illinois: Rand McNally, 1996.

DEAN, Angela M.; VOSS, Daniel. Design and analysis of experiments. New York: Springer, 1999.

DELLA PORTA, Donatella; KEATING, Michael (Ed.). Approaches and methodologies in the social sciences: a pluralist perspective. Cambridge: Cambridge University Press, 2008. 
ELSTER, Jon. Nuts and bolts for the social sciences. Cambridge: Cambridge University Press, 1989.

FIGUEIREDO FILHO, Dalson; SILVA JUNIOR, José Alexandre; ROCHA, E. 0 que fazer e 0 que não fazer com a regressão: pressupostos e aplicações do modelo linear de mínimos quadrados ordinários (MQ0). Política Hoje, Recife, v. 20, 1, 44-99, 2011.

FRIEDRICH, Robert J. In defense of multiplicative terms in multiple regression equations. American Journal of Political Science, Austin, v. 26, n. 4, p. 797-833, 1982.

GERRING, John. Social science methodology: a criterial framework. Cambridge: Cambridge University Press, 2001.

. Causation: a unified framework for the Social Sciences. Iournal of Theoretical Politics, London, v. 17, n. 2, p.163-98, 2005.

HEDSTRÖM, Peter; SWEDBERG, Richard. Social mechanisms: an introductory essay. In: Social mechanisms: an analytical approach to social theory. New York: Cambridge University Press, 1998.

HOLLAND, Paul. Statistics and causal inference. Journal of the American Statistical Association, Baltimore, v. 81, p. 945-60, 1986.

JACCARD, James; TURRISI, Robert; WAN, Choi. Interaction efects in multiple regression. Newbury Park, CA: Sage, 1990.

KING, Gary; KEOHANE, Robert; VERBA, Sidney. Designing social inquiry: scientific inference in qualitative research. Princeton: Princeton University Press, 1994.

KITSCHELT, Hebert. Accounting for postcommunist regime diversity: what counts as a good cause? In: EKIERT, Grzegorz; HANSON, Stephen E. (Ed.). Capitalism and democracy in central and eastern europe. Cambridge: Cambridge University Press, 2003. p. 49-86.

KUHH, Thomas. A estrutura das revoluções cientificas. 3. ed. São Paulo: Perspectiva, 1975.

MAHONEY, James. Path-Dependent explanations of regime change: Central America in comparative perspective. Studies in Comparative International Development, Sant Louis, v. 36, p. 111-141, 2001.

MARSH, David; FURLONG, Paul. A skin not a sweater: ontology and epistemology in political science. In: MARSH, David; STOKER, Gerry. (Ed.). Theory and methods in political science. Basingstoke: Palgrave, 2002. p. 17-44.

MERTON, R. Social theory and social structure. NewYork: The Free Press, 1968. 
MONTGOMERY, Douglas. Design and analysis of experiments. $5^{\text {th }}$ edn. New York: John Wiley \& Sons, 2001.

PEARL, Judea. Causality: models, reasoning, and inference. New York: Cambridge University Press, 2000.

RATTON JUNIOR, Jose Luiz; MORAIS, Jorge Ventura de. Para ler Jon Elster: limites e possibilidades da explicação por mecanismos nas Ciências Sociais. DADOS - Revista de Ciências Sociais, Rio de Janeiro, v. 46, n. 2, p. 385-410, 2003.

RUBIN, Donald B. Estimating causal efects of treatments in randomized and nonrandomized studies. Journal of Educational Psychology, Arlington, v. 66, n. 5, p. 688-701, 1974.

SALMON, Wesley. Scientific explanation: three basic conceptions. Proceedings of the biennial Meeting of the Philosophy of Science Association. Symposia and Invited Papers, Chicago, v. 2, p. 293-305, 1984.

SANDERS, David. Behaviouralism. In: MARSH, David; STOKER, Gerry (Ed.). Theory and methods in political science. Basingstoke: Palgrave, 2002. p. 45-64.

SHADISH, R.; COOK, T.; CAMPBELL. Experimental and quasi-experimental Designs of generalized causal inference. New York: Houghton Mifflin Company, 2002.

STINCHCOMBE, Arthur. The conditions of fruitfulness of theorizing about mechanisms in Social Science. Philosophy of the Social Sciences, Waterloo, v. 21, n. 3, p. 367-388, 1991.

TILLY, Charles. Mechanisms in political process. Annual Review of Political Science, Palo Alto, v. 4, p. 21-41, 2001.

VAN EVERA, Stephen. Guide to methods for students of political science. Ithaca, NY: Cornell University Press, 1997.

ZALD, Mayer N. Progress and cumulation in the human sciences after the fall. Sociological Forum, New York, v. 10, n. 3, p. 455-479, 1995. 